\title{
Antibacterial and cytotoxic properties of isoprenoids from the red sea soft coral, Lobophytum sp
}

\author{
Khalid O Al-Footy ${ }^{1}$, Walied M Alarif ${ }^{2 *}$, Muhammad S Zubair ${ }^{1,3}$, Mohamed A \\ Ghandourah $^{2}$ and Magda M Aly ${ }^{4}$ \\ ${ }^{1}$ Department of Chemistry, Faculty of Science, ${ }^{2}$ Department of Marine Chemistry, Faculty of Marine Sciences, King Abdulaziz \\ University, PO Box 80207, Jeddah 21589, Saudi Arabia, ${ }^{3}$ Department of Pharmacy, Faculty of Science, Tadulako University, \\ Kampus Bumi Tadulako Tondo, Palu 94118, Indonesia, ${ }^{4}$ Department of Biology, Faculty of Science, King Abdulaziz University, \\ PO. Box 80203, Jeddah 21589, Saudi Arabia
}

*For correspondence: Email: walied1737@yahoo.com, welaref@kau.edu.sa; Tel: +966-56-0352034

\begin{abstract}
Purpose: To evaluate the antibacterial and cytotoxic activities of the secondary metabolites of Lobophytum $s p$.

Methods: Maceration with methanol: chloroform (1:1) was applied to extract the coral material. Chromatographic and spectroscopic techniques were employed for fractionation, isolation and elucidation of pure compounds. Antibacterial activities were performed by well diffusion method against three Gram-positive and four Gram-negative bacteria. Brine shrimp lethality test was employed to predict toxicity, while antitumor activity were tested by 3-(4, 5-dimethylthiazol-2-yl)-2, 5diphenyltetrazolium bromide (MTT) method against Ehrlich carcinoma cells.

Results: Four sesquiterpenes, one cembranoid type diterpenes and two steroids were isolated. 1 exhibited significant antibacterial activity against four tested bacteria ( $P$. aeruginosa, S. aureus, S. epidermis, and S. pneumonia) with MIC value of $15 \mu \mathrm{g} / \mathrm{mL}$. Moreover, 1 showed high diameter zone of inhibition ranging from $16-18 \mathrm{~mm}$ against test bacteria. Compounds 4 and 5 displayed moderate antibacterial activity against all test bacteria with inhibition zone diameter (IZD) ranging from $11-15$ $\mathrm{mm}$ and MIC values of $30 \mu \mathrm{g} / \mathrm{mL}$. 2, 3, 6 and 7 exhibited weak antibacterial activity (IZD, $7-11 \mathrm{~mm}$; $M I C \geq 30 \mu \mathrm{g} / \mathrm{mL}$ ). In addition, only diterpene compound (4) showed high toxicity against A. Salina and antitumor activity against Erhlich carcinoma cells with the $L D_{50}$ of 25 and $50 \mu \mathrm{g} / \mathrm{mL}$, respectively.

Conclusion: This study reveals the strong antibacterial activity of sesquiterpene alismol (1) and the potential antibacterial and antitumor activity of cembranoid type diterpene, cembrene A (4).
\end{abstract}

Keywords: Soft coral, Lobophytum sp., Red Sea, Antibacterial, Cytotoxicity, Sesquiterpene Alismol, Cembranoid, Diterpene, Cembrene

Tropical Journal of Pharmaceutical Research is indexed by Science Citation Index (SciSearch), Scopus, International Pharmaceutical Abstract, Chemical Abstracts, Embase, Index Copernicus, EBSCO, African Index Medicus, JournalSeek, Journal Citation Reports/Science Edition, Directory of Open Access Journals (DOAJ), African Journal Online, Bioline International, Open-J-Gate and Pharmacy Abstracts

\section{INTRODUCTION}

Marine soft corals of the subclass Alcyonaria (Octocorallia) have fascinated a great attention in the light of the structural diversity and broad range of biological activities of their metabolites, such as sesquiterpenoids, diterpenoids, tetraterpenoids and steroids [1]. In a continuing efforts at studying marine metabolites, collected from Red Sea Saudi Arabia, with biological activity, we have previously isolated several bioactive secondary metabolites from Sarcophyton trocheliophorum and S. glaucum [25] Recently, our interest in allcyonaria coral, led us to isolate several secondary metabolites from Lobophytum sp. Literature survey showed that 
metabolites of Lobophytum sp exhibited various biological activities, such as antitumor [6-13], antibacterial [14], anti-inflammatory activities [1416] and antiviral [17]. Our investigation led to the isolation and characterization of four sesquiterpenes: alismol (1), nardol (2) aristol-9ene (3), alismoxide (5), one cembranoid type diterpene, cembrene A (4), and two steroid type compounds, Chalinasterol (6) and Nephalsterol $C$ (7). To the best of our knowledge, this is the first report of the presence of sesquiterpenes from soft coral Lobophytum sp. Antibacterial and antitumor activities of all metabolites were also evaluated. This paper describes isolation, NMR structural elucidation and biological activity of the following compounds.

\section{EXPERIMENTAL}

\section{Animal material}

The soft coral, Lobophytum sp., was collected in May. 2014, off the Saudi Arabia Red Sea Coast at Jeddah and identified by Dr. Mohsen ElSherbiny (Marine Biology Department, Faculty of Marine Sciences, King Abdulaziz University). Voucher sample (no. JAD 09063B) was deposited in the herbarium of the Marine Biology Department, Faculty of Marine Sciences, King Abdulaziz University, Jeddah, Saudi Arabia

\section{General experimental procedures}

Chromatographic material: Silica gel 60 for column chromatography (60-120 mesh LR), for thin-layer chromatography. TLC aluminum sheets $20 \times 20 \mathrm{~cm}$. Silica gel 60 F254 was used for preparative, Pre-coated TLC glass plates SIL G-25 UV254, $0.25 \mathrm{~mm}$ silica gel (E. Merck, Darmstadt, Germany). Nuclear Magnetic Resonance spectra were recorded for 1D- and 2D-NMR: Bruker AVANCE III WM at $600 \mathrm{MHz}$ and ${ }^{13} \mathrm{C}-\mathrm{NMR}$ at $150 \mathrm{MHz}$ spectrometer. Chemical shifts are given $d(p p m)$ relative to TMS as internal standard. Electron impact mass spectra were determined at $70 \mathrm{eV}$ using a Kratos GCMS-25 instrument. All chemicals were purchased from Sigma Chemical Company (St. Louis, MO. USA).

\section{Bacterial isolates}

Seven bacteria were obtained from King Fahd General Hospital, Jeddah, Saudi Arabia; they were Staphylococcus aureus, Staphylococcus epidermidis, and Streptococcus pneumonia (Gram-positive bacteria), Pseudomonas aeruginosa, Escherichia coli, Klebsiela pneumoniae, and Acinetobacter spp (Gramnegative bacteria), Brine shrimp Artemia salina, and Ehrlich carcinoma cells were from the culture collection of Microbiology Department, Faculty of Science, King Abdulaziz University, Jeddah, Saudi Arabia.

\section{Extraction and isolation}

Coral material was washed with water and dried in the shade at room temperature. The dried material $(90 \mathrm{~g})$ was exhaustively extracted by maceration method with equal volumes of $\mathrm{CHCl}_{3} / \mathrm{MeOH}(2 \times 6 \mathrm{I}, 24 \mathrm{~h}$ for each batch) at room temperature.
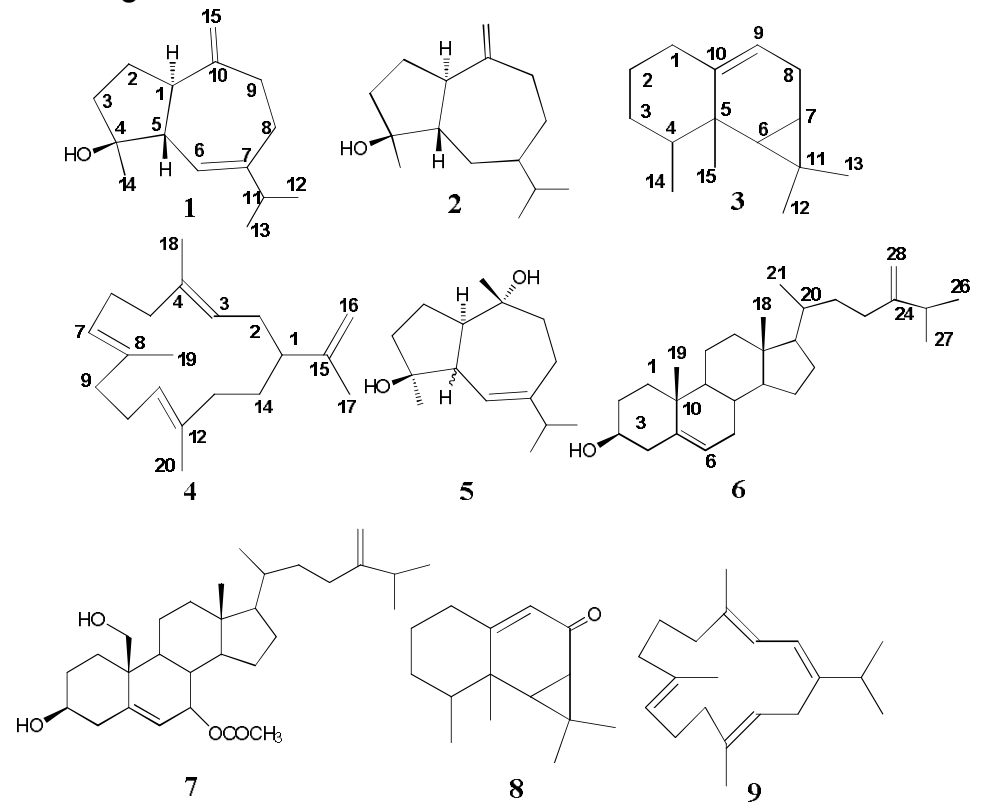
6

Figure 1: Chemical structures of 1 - 9 
The residue $(20 \mathrm{~g})$ was chromatographed on NP (Merck, 60G) column chromatography employing n-hexane/methylene chloride, followed by ethyl acetate/methanol mixtures with increasing polarity. Fractions of $\sim 100 \mathrm{~mL}$ were collected. TLC was carried out by employing silica gel chromatoplates, appropriate solvent system, and $50 \%$-sulfuric acid in methanol as spraying reagent. Fractions containing a single compound were combined and further purified by preparative TLC of glass supported silica gel plates $(20 \times 20 \mathrm{~cm})$ of $250 \mu \mathrm{m}$ thickness.

The fraction 1 eluted with n-hexane dichloromethane $(9: 1,37.00 \mathrm{mg})$ was purified by preparative TLC using solvent system n-hexane: ethyl acetate $(9: 1)$ to give two bands. The first band with $R_{f}=0.96$ (brown color with sulfuric acid-methanol) was taken to give compound $\mathbf{3}$ as crystalline $(5.0 \mathrm{mg})$. The second band with $R_{f}$ $=0.36$ (black color with sulfuric acid-methanol) was taken to give compound $\mathbf{4}$ as colorless oil $(4.8 \mathrm{mg})$. The fraction 2 eluted with $\mathrm{n}$-hexane dichloromethane $(7: 3,35.00 \mathrm{mg})$ was purified by preparative TLC using the solvent system nhexane:ethyl acetate $(8: 2)$ to give two bands. The first band with $R_{f}=0.48$ (broad purple color after spraying with sulfuric acid-methanol) was taken to give crystalline of compound $1(6.5 \mathrm{mg})$. The second band with $R_{f}=0.30$ (purple color with sulfuric acid-methanol) was taken to give colorless oil of compound 2 (4.6 mg). The fraction 3 eluted with n-hexane dichloromethane $(5: 5,40.00 \mathrm{mg})$ was purified by preparative TLC using solvent system n-hexane: ethyl acetate $(7: 3)$ to give one band with $R_{f}=$ 0.72 (brown color with sulfuric acid-methanol) was taken to give compound 6 as crystalline (5.0 $\mathrm{mg})$. The fraction 4 eluted with dichloromethane:ethyl acetate $(9: 1,42.00 \mathrm{mg})$ was purified by preparative TLC using the solvent system n-hexane : ethyl acetate (6:4) to give one band with $R_{f}=0.38$ (broad purple color after spraying with sulfuric acid-methanol) was taken to give crystalline of compound $5(8.0 \mathrm{mg})$. The fraction 5 eluted with dichloromethane:ethyl acetate $(8: 2,41.00 \mathrm{mg})$ was purified by preparative TLC using the solvent system nhexane:ethyl acetate $(5: 5)$ to give one band with $R_{f}=0.62$ (brown color after spraying with sulfuric acid-methanol) was taken to give powder of compound 7 (4.2 mg).

\section{Antibacterial activity assay}

The growth inhibition of the isolated compounds against several pathogenic bacteria was carried out using agar well diffusion method as described by Holder and Boyce (1994) [18]. 0.1 $\mathrm{mL}$ of suspended bacterium in sterile medium $\left(1.5 \times 10^{8} \mathrm{CFU} / \mathrm{mL}\right)$ was spread on MuellerHinton agar. $50 \mu \mathrm{l}$ of each sample $(10 \mu \mathrm{g} / \mathrm{mL})$ were poured to the wells (6-mm diameter). All plates were left for $1 \mathrm{~h}$ at $48{ }^{\circ} \mathrm{C}$ and then incubated for $24 \mathrm{~h}$ at $37{ }^{\circ} \mathrm{C}$. Inhibition zone diameters formed around the well were measured and mean diameter of three replica was calculated. DMSO was used as a negative control and ampicillin as a positive control. Minimum inhibitory concentrations (MICs) were determined by modified method described by Chand et al [19] and Aly and Gumgumji [20]. 175 $\mu \mathrm{L}$ of an exponentially growing culture $\left(\times 10^{6}-10^{7}\right.$ $\mathrm{CFU} / \mathrm{mL}$ ) was delivered into each well of microtiter plate along with $20 \mu \mathrm{L}$ solution of each concentration of the isolated compounds. The appropriate solvent was used as control. After incubation for $40 \mathrm{~min}$., $5 \mu \mathrm{L}$ of a solution of Fluorescein diacetate (FDA) $0.2 \%(\mathrm{w} / \mathrm{v})$ in acetone was added into microtiter plate, then the incubation was continued for $90 \mathrm{~min}$. The green color produced from the hydrolysis of FDA was measured at $490 \mathrm{~nm}$ (MR7000 automatic ELISA tray reader) along with the blanked wells and control wells containing microbial cultures.

\section{Toxicity activity assay}

The cytotoxicity of the isolated compounds can be measured by using brine shrimp lethality test [21]. Isolated compounds were dissolved in DMSO at varying concentrations and then incubated with the brine shrimp larvae in sea water. Brine shrimp larvae as positive control and DMSO as negative control were also incubated. The average number of larvae that survived in each vial was determined after $24 \mathrm{~h}$. The mean of mortality percentage was plotted against the logarithm of concentrations. The concentration that killed fifty percent of the larvae $\left(\mathrm{LC}_{50}\right)$ was determined from the graph.

\section{Antitumor activity assay}

Ehrlich carcinoma cell lines was used to determine the antitumor activity of the isolated compounds. The cells were grown in Roswell Park Memorial Institute (RPMI) 1640 medium (Sigma, USA) with $10 \%$ fetal calf serum (Gibco, USA) at $37{ }^{\circ} \mathrm{C}$ under a humidified atmosphere $\left(95 \%\right.$ air and $5 \% \mathrm{CO}_{2}$ ) for $48 \mathrm{~h}$. Cells were treated with different concentration of the isolated compounds $(200-1,000 \mu \mathrm{l} / \mathrm{mL})$ for $24 \mathrm{~h}$, centrifuged for $2 \mathrm{~min}$ at $1,500 \mathrm{~g}$ and counted under hemacytometer after removing the supernatant using trypan blue (Sigma, USA) in normal saline $(1: 1 \mathrm{v} / \mathrm{v})$. The percentage of cell viability was assessed to determine the $50 \%$ lethal dose by which $50 \%$ of cells are killed $\left(\mathrm{LD}_{50}\right)$. 


\section{Statistical analysis}

All experiments were performed in triplicate. Data are presented as mean \pm standard deviation (SD). Unpaired student t-test was carried out to detect any significant differences between the results of control and the treated sampels using GraphPad InStat (ISI Software). Differences were considered significant at $p<0.05$.

\section{RESULTS}

\section{Compounds}

Identification of secondary metabolites was analyzed based on MS and 1D/2D NMR data and comparison with the literature. The chemical structure of isolated compounds can be seen in the Fig. 1.

Alismol (1): Colorless oil; HRESIMS: $m / z=220.3505$ (Calc. for $\mathrm{C}_{15} \mathrm{H}_{24} \mathrm{O}, 220.1820$ ); ${ }^{1} \mathrm{H}$ NMR (600 MHz, CDCl $): \delta_{\mathrm{H}} 5.55(1 \mathrm{H}$, br s, H-6), $4.76(1 \mathrm{H}, \mathrm{s}, \mathrm{Ha}-15), 4.70(1 \mathrm{H}, \mathrm{s}, \mathrm{Hb}-15), 2.28$ $(1 \mathrm{H}, \mathrm{dd}, \mathrm{J}=14.4,7.4 \mathrm{~Hz}, \mathrm{H}-5), 2.50$ (dd, $\mathrm{J}=21.6$, $10.2 \mathrm{~Hz}, \mathrm{H}_{1}-1$ ), 2.06 (dd, J=38.4, $10.2 \mathrm{~Hz}, \mathrm{H}_{2}-1$ ), $2.30(1 \mathrm{H}, \mathrm{d}, \mathrm{J}=7.4 \mathrm{~Hz}, \mathrm{H}-1), 2.24(1 \mathrm{H}, \mathrm{m}, \mathrm{H}-11)$, $2.20(1 \mathrm{H}, \mathrm{m}, \mathrm{Ha}-8), 2.10(1 \mathrm{H}, \mathrm{m}, \mathrm{Hb}-8), 1.90(1 \mathrm{H}$, $\mathrm{m}, \mathrm{H}-2), 1.74(2 \mathrm{H}, \mathrm{m}, \mathrm{H}-3), 1.72(1 \mathrm{H}, \mathrm{m}, \mathrm{H}-2$ '), $1.25(3 \mathrm{H}, \mathrm{s}, \mathrm{H}-14), 1.01(3 \mathrm{H}, \mathrm{d}, J=6.6 \mathrm{~Hz}, \mathrm{H}-$ 12), $0.98(3 \mathrm{H}, \mathrm{d}, J=6.6 \mathrm{~Hz}, \mathrm{H}-13) ;{ }^{13} \mathrm{C} \mathrm{NMR}$ $\left(200 \mathrm{MHz}, \mathrm{CDCl}_{3}\right): \delta_{\mathrm{C}} 153.93(\mathrm{C}-10), 149.78(\mathrm{C}-$ 7), 121.30 (C-6), 106.51 (C-15), 80.69 (C-4), 55.01 (C-1), 47.27 (C-5), 40.25 (C-3), 37.44 (C11), 37.08 (C-9), 30.00 (C-8), 24.76 (C-2), 24.09 (C-14), 21.48 (C-13), 21.30 (C-12).

Nardol (2): Colorless Oil; HRESIMS: $m / z=222.3663$ (Calc. for $\mathrm{C}_{15} \mathrm{H}_{26} \mathrm{O}, 222.1984$ ); ${ }^{1} \mathrm{H}$ NMR (600 MHz, CDCl $\left.{ }_{3}\right): \delta \mathrm{H} 4.73\left(1 \mathrm{H}, \mathrm{s}, \mathrm{H}-15^{\prime}\right)$, $4.71(1 \mathrm{H}, \mathrm{s}, \mathrm{H}-15), 2.89(1 \mathrm{H}, \mathrm{d}, \mathrm{H}-1), 2.40(1 \mathrm{H}$, $\mathrm{m}, \mathrm{H}-9), 2.30(1 \mathrm{H}, \mathrm{m}, \mathrm{H}-7), 2.16(1 \mathrm{H}, \mathrm{m}, \mathrm{H}-8)$, $2.14(1 \mathrm{H}, \mathrm{m}, \mathrm{H}-9$ '), $1.94(1 \mathrm{H}, \mathrm{m}, \mathrm{H}-6), 1,84(1 \mathrm{H}$, m, H-3), 1.72 (1H, m, H-6'), $1.71(1 \mathrm{H}, \mathrm{m}, \mathrm{H}-5)$, $1.62\left(1 \mathrm{H}, \mathrm{m}, \mathrm{H}-3^{\prime}\right), 1.58(1 \mathrm{H}, \mathrm{m}, \mathrm{H}-11), 1.52(1 \mathrm{H}$, m, H-8'), 1.44 (3H, s, H-14), $1.30(2 \mathrm{H}, \mathrm{m}, \mathrm{H}-2)$, $1.01(3 \mathrm{H}, \mathrm{d}, J=6.6 \mathrm{~Hz}, \mathrm{H}-12), 0.96(3 \mathrm{H}, \mathrm{d}, J=$ 6.6 Hz, H-13); ${ }^{13} \mathrm{C}$ NMR $\left(200 \mathrm{MHz} \mathrm{CDCl}_{3}\right): \delta \mathrm{C}$ 151.63 (C-10), 107.28 (C-15), 80.31 (C-4), 62.18 (C-1), 57.57 (C-5), 47.91 (C-7), 41.50 (C-3), 34.58 (C-11), 34.06 (C-9), 29.15 (C-8), 26.27 (C6), 24.95 (C-14), 22.71 (C-2), 18.72 (C-13), $17.74(\mathrm{C}-12)$.

Aristol-9-ene (3): Colorless oil; HRESIMS: $m / z=$ 204.3511 (Calc. for $\mathrm{C}_{15} \mathrm{H}_{24}, 204.1878$ ); ${ }^{1} \mathrm{H}$ NMR $\left(600 \mathrm{MHz}, \mathrm{CDCl}_{3}\right): \delta_{\mathrm{H}} 5.10(1 \mathrm{H}, \mathrm{ddd}, 2.4,2.4,4.2$ $\mathrm{Hz}, \mathrm{H}-9), 2.16(1 \mathrm{H}, \mathrm{m}, \mathrm{H}-1), 1.98(1 \mathrm{H}, \mathrm{m}, \mathrm{H}-1)$, $2.08(1 \mathrm{H}, \mathrm{dd}, 2.4,4.2$, Ha-8), $2,04(1 \mathrm{H}, \mathrm{dd}, 2.4$, 4.2, Hb-8), $1.69(1 \mathrm{H}, \mathrm{dd}, 1.2,2.4, \mathrm{H}-4), 1.62(\mathrm{H}$, m, Ha-3), 1.42 (1H, m, Ha-2), 1.36 (1H, m, Hb-3), $1.3(1 \mathrm{H}, \mathrm{m}, \mathrm{Hb}-2), 1.18(3 \mathrm{H}, \mathrm{s}, \mathrm{H}-13), 1.12(3 \mathrm{H}$, s, H-12), $1.05(3 \mathrm{H}, \mathrm{s}, \mathrm{H}-15), 0.98(3 \mathrm{H}, \mathrm{d}, \mathrm{J}=4.8$ $\mathrm{Hz}, \mathrm{H}-14), 0.75(1 \mathrm{H}, \mathrm{dd}, \mathrm{J}=6,8.5 \mathrm{~Hz}, \mathrm{H}-7), 0.60$ $(1 \mathrm{H}, \mathrm{d}, J=8.5 \mathrm{~Hz}, \mathrm{H}-6) ;{ }^{13} \mathrm{C} \mathrm{nmr}(200 \mathrm{MHz}$, $\left.\mathrm{CDCl}_{3}\right): \delta_{\mathrm{C}} 141.30(\mathrm{C}-10), 118.5(\mathrm{C}-9), 37.86$ (C4), 36.82 (C-5), 33.04 (C-1), 32.09 (C-6), 31.32 (C-2), 28.44 (C-7), 27.21 (C-3) 21.77 (C-8), 21.29 (C-13), 19.29 (C-14), 17.9 (C-11), 15.91 $(\mathrm{C}-12), 15.65$ (C-15).

Cembrene A (4): Colorless oil, HRESIMS: $m / z=$ 272.4681 (Calc.for $\mathrm{C}_{20} \mathrm{H}_{32}, 272.2504$ ); ${ }^{1} \mathrm{H}$ NMR $\left(600 \mathrm{MHz} \mathrm{CDCl}_{3}\right): \delta_{\mathrm{H}} 5.31(1 \mathrm{H}, \mathrm{dd}, J=6.8,15.3$ $\mathrm{Hz}, \mathrm{H}-7), 5.25(1 \mathrm{H}, \mathrm{dt}, \mathrm{J}=1.7,6.8,8.5 \mathrm{~Hz}, \mathrm{H}-3)$, $5.08(1 \mathrm{H}, \mathrm{dt}, \mathrm{J}=6.0,12.0, \mathrm{H}-11), 4.82(2 \mathrm{H}, \mathrm{d}$, $J=1.0, \mathrm{H}-16), 2.26,(1 \mathrm{H}, \mathrm{m}, \mathrm{Ha}-6), 2.21(1 \mathrm{H}$, dddd, J=3.4, 4.25, 10.2, $13.6 \mathrm{~Hz}, \mathrm{H}-1), 2.17(1 \mathrm{H}$, m, Ha-2), 2.15 (2H, m, H-10), 2.15 (1H, m, Ha-5), $2.13(1 \mathrm{H}, \mathrm{m}, \mathrm{Hb}-6), 2.12$ (1H, m, Ha-13), 2.09 $(2 \mathrm{H}, \mathrm{m}, \mathrm{H}-9), 2.02(1 \mathrm{H}, \mathrm{m}, \mathrm{Hb}-2), 1.96(1 \mathrm{H}, \mathrm{m}$, $\mathrm{Hb}-5), 1.93$ (1H, m, Hb-13), 1.79 (dq, $J=10.2$; 13.6 Hz, Ha-14), 1.41 (m, Hb-14), 1.63 (3H, s, H17), $1.60(3 \mathrm{H}, \mathrm{s}, \mathrm{H}-18), 1.55(3 \mathrm{H}, \mathrm{s}, \mathrm{H}-19), 1.54$ $(3 \mathrm{H}, \mathrm{s}, \mathrm{H}-20) ;{ }^{13} \mathrm{C}$ NMR $\left(150 \mathrm{MHz}, \mathrm{CDCl}_{3}\right) ; \delta_{\mathrm{C}}$ 148.87 (C-15), 134.55 (C-8), 133.48 (C-4), 133.10 (C-12), 126.08 (C-11), 124.19 (C-7), 121.91 (C-3), 110.44 (C-16), 46.17 (C-1), 39.49 (C-9), 39.01 (C-5), 33.99 (C-13), 32.64 (C-2), 28.19 (C-14), 24.98 (C-6), 23.85 (C-10), 18.99 (C-17), 17.97 (C-18), 15.5 (C-19), 15.28 (C-20).

Alismoxide (5): Colorless oil, HRESIMS: $\mathrm{m} / \mathrm{z}=$ 238.3657 (Calc. for $\mathrm{C}_{15} \mathrm{H}_{26} \mathrm{O}_{2}, 238.1930$ ), ${ }^{1} \mathrm{H}$ NMR (600 MHz, CDCl $\left.)_{3}\right): \delta_{H} 5.50(1 \mathrm{H}, \mathrm{dd}, J=2.0$; $1.2 \mathrm{~Hz}, \mathrm{H}-6), 2.24(1 \mathrm{H}$, sept, $\mathrm{H}-11), 2.22(1 \mathrm{H}, \mathrm{m}$, Ha-8), 2.18 (1H, m, H-5), 1.95 (1H, dd, 10.4; 1.2 $\mathrm{Hz}, \mathrm{Hb}-8), 1.89$ (1H, m, H-1), 1.80 (1H, m, Ha-2), $1.80(1 \mathrm{H}, \mathrm{m}, \mathrm{Ha}-9), 1.70(1 \mathrm{H}, \mathrm{m}, \mathrm{Ha}-3), 1.68(1 \mathrm{H}$, $\mathrm{m}, \mathrm{Hb}-2), 1.62$ (1H, m, Hb-3), 1.47 (1H, m, Hb-9), $1.27(3 \mathrm{H}, \mathrm{s}, \mathrm{H}-14), 1.21(3 \mathrm{H}, \mathrm{s}, \mathrm{H}-15), 0.99(3 \mathrm{H}$, d, $J=6.8 \mathrm{~Hz}, \mathrm{H}-12), 0.98(3 \mathrm{H}, \mathrm{J}=6.8 \mathrm{~Hz}, \mathrm{H}-13)$; ${ }^{13} \mathrm{C}$ NMR (150 MHz, CDCl 3 ): $\delta_{\mathrm{C}} 149.69$ (C-7), 121.33 (C-6), 80.25 (C-4), 75.25 (C-10), 50.77 (C-1), 50.38 (C-5), 42.67 (C-9), 40.52 (C-3), 37.33 (C-11), 25.12 (C-8), 21.44 (C-14), 21.55 (C-2), 22.61 (C-15), 21.20 (C-13), 21.42 (C-12).

Chalinasterol (6): Amorphous powder, HRESIMS: $m / z=398.6642$ (Calc. for $\mathrm{C}_{28} \mathrm{H}_{46} \mathrm{O}$, 398.3540), ${ }^{1} \mathrm{H}$ NMR (600 MHz, $\left.\mathrm{CDCl}_{3}\right) ; \delta_{\mathrm{H}} 5.38$ $(1 \mathrm{H}, \mathrm{s}, \mathrm{H}-6), 4.71(1 \mathrm{H}, \mathrm{s}, \mathrm{Ha}-28), 4.65(1 \mathrm{H}, \mathrm{s}, \mathrm{Hb}-$ 28), $3.6(1 \mathrm{H}, \mathrm{m}, \mathrm{H}-3), 1.03(3 \mathrm{H}, \mathrm{d}, J=6.8 \mathrm{~Hz}, \mathrm{H}-$ 27), 1.02 (3H, d, J=6.8 Hz, H-26'), 1.01 (3H, s, H19), $0.98(3 \mathrm{H}, \mathrm{d}, J=6.8 \mathrm{~Hz}, \mathrm{H}-26), 0.94(3 \mathrm{H}, \mathrm{d}, \mathrm{J}=$ 6.8, H-21), $0.68(3 \mathrm{H}, \mathrm{s}, \mathrm{H}-18) .{ }^{13} \mathrm{C}$ NMR (150 $\left.\mathrm{MHz}, \mathrm{CDCl}_{3}\right): \delta_{\mathrm{C}} 156.98(\mathrm{C}-24), 140.79$ (C-5), 121.73 (C-6), 105.91 (C-28), 71.83 (C-3), 56.77 (C-14), 55.99 (C-17), 50.13 (C-9), 42.37 (C-13), 42.31 (C-4), 39.79 (C-12), 37.48 (C-1), 36.51 (C- 
10), 35.7 (C-20), 34.69 (C-23), 33.81 (C-25), 38.58 (C-8), 32.0 (C-7), 31.68 (C-2), 30.98 (C22), 28.23 (C-16), 24.3 (C-15), 22.01 (C-27), 21.88 (C-26), 21.10 (C-11), 19.42 (C-19), 18.72 (C-21), 11.88 (C-18).

Nephalsterol C (7): Amorphous powder, HRESIMS: $m / z=472.6997$ (Calc. for $\mathrm{C}_{30} \mathrm{H}_{48} \mathrm{O}_{4}$, 472.3550), ${ }^{1} \mathrm{H}$ NMR (600 MHz, $\left.\mathrm{CDCl}_{3}\right): \delta \mathrm{H} 5.58$ $(1 \mathrm{H}, \mathrm{t}, J=2 \mathrm{~Hz}, \mathrm{H}-6), 4.90(1 \mathrm{H}, \mathrm{dd}, J=2.5,6 \mathrm{~Hz}$, $\mathrm{H}-7), 4.71(1 \mathrm{H}, \mathrm{d}, J=0.85 \mathrm{~Hz}, \mathrm{Ha}-28), 4.65(1 \mathrm{H}$, $\mathrm{d}, J=0.85 \mathrm{~Hz}, \mathrm{Hb}-28), 3.80(1 \mathrm{H}, \mathrm{d}, J=11.9 \mathrm{~Hz}$, $\mathrm{H}-19), 3.65$ (1H, d, J=11.9 Hz, H-19'), 3.60 (1H, $\mathrm{tt}, J=10.25 .1 \mathrm{~Hz}, \mathrm{H}-3), 2.38(1 \mathrm{H}$, dddd, $J=1.7$, 2.5, 4.25, $5.95 \mathrm{~Hz}, \mathrm{H}-4), 2.20(1 \mathrm{H}, \mathrm{tt}, 6.8,12.75$, $\mathrm{H}-4$ ') $2.02(3 \mathrm{H}, \mathrm{s}, \mathrm{OAc}), 1.02(3 \mathrm{H}, \mathrm{d}, J=5.1 \mathrm{~Hz}$, $\mathrm{H}-26), 1.01(3 \mathrm{H}, \mathrm{d}, J=5.1 \mathrm{~Hz}, \mathrm{H}-27), 0.94(3 \mathrm{H}$, $\mathrm{d}, J=5.9 \mathrm{~Hz}, \mathrm{H}-21), 0.74(3 \mathrm{H}, \mathrm{s}, \mathrm{H}-18) .{ }^{13} \mathrm{C} \mathrm{NMR}$ (150 MHz, CDCl3): $\delta_{\mathrm{C}} 171.38$ (MeCOO-), 156.81 (C-24), 140.02 (C-5), 126.78 (C-6), 106.02 (C28), 75.11 (C-7), 70.92 (C-3), 62.9 (C-19), 56.33 (C-14), 55.27 (C-17), 48.53 (C-9), 42.6 (C-13), 41.63 (C-4), 41.45 (C-10), 39.74 (C-12), 35.65 (C-8), 37.84 (C-20), 34.65 (C-22), 33.78 (C-25), 33.20 (C-1), 31.80 (C-2), 30.95 (C-23), 28.39 (C16), 24.98 (C-15), 21.73 (C-26), 21.86 (C-27), $21.7\left(\mathrm{CH}_{3} \mathrm{COO}\right), 22.01$ (C-11), 18.74 (C-21), 12.13 (C-18).

\section{Antibacterial activity}

Antibacterial activity assay were performed against several bacteria. The result of diameter of zone of inhibitons $(\mathrm{mm})$ of isolated compounds were presented in Table 1. There were significant differences $(p<0.05)$ among antibacterial activities of all isolated compounds. Compound 1 has significant antibacterial activity $(p<0.05)$ against both gram-positive bacteria $(S$. aureus, S. S. epidermis and $S$. pneumonia) and gram-negative bacteria ( $P$. aeruginosa) with the MIC value of $15 \mu \mathrm{g} / \mathrm{mL}$ (Table 2). Moreover, compound 1 showed the larger diameter of inhibition zone ranging from $16-18 \mathrm{~mm}$ against those four bacteria. The diameter of inhibition zone against $S$. epidermis was recorded to be largest (18 $\pm 2.0 \mathrm{~mm})$. Meanwhile, compound 4 and 5 exhibited moderate antibacterial activity with the diameter of inhibiton zone ranging from $11-15 \mathrm{~mm}$ and MIC value of $30 \mu \mathrm{g} / \mathrm{mL}$. Compound 2, 3, 6 and 7 showed weak antibacterial activity with diameter of inhibition zone ranging from $7-10 \mathrm{~mm}$ and $\mathrm{MIC}$ value $\geq$ $30 \mu \mathrm{g} / \mathrm{mL}$.

\section{Toxicity and antitumor activity}

The result of toxicity and antitumor activity test can be seen in Table 3. All compounds did not show appreciable toxicity and antitumor properties against Artemia salina and Erhlich carcinoma cells, except for cembrene A (compound 4). It exhibited significant toxicity and antitumor activity $(p<0.05)$ with the $L_{50}$ values of 25 and $50 \mu \mathrm{g} / \mathrm{mL}$, respectively.

\section{DISCUSSION}

Compound 1 was isolated as colorless oil. HRESIMS at $\mathrm{m} / \mathrm{z}$ of 220.3505 and ${ }^{13} \mathrm{C}$ NMR suggesting a molecular formula of $\mathrm{C}_{15} \mathrm{H}_{24} \mathrm{O}$ with 4 degrees of unsaturation. The obtained spestroscopic data (cf. exp.) was found to be identical with the structural data of alismol, a guaiane sesquiterpene isolated previously from the rhizome of Alisma plantago-aquatu $\mathrm{L}$ var orlentale Samuelsson (Altsmataceae) [22,23].

Table 1: Antibacterial activity of compounds 1-7

\begin{tabular}{|c|c|c|c|c|c|c|c|c|}
\hline \multirow{2}{*}{ Test bacteria } & \multicolumn{8}{|c|}{ Mean diameter of the inhibition zone $(\mathrm{mm}) \pm \mathrm{SD}^{*}$} \\
\hline & 1 & 2 & 3 & 4 & 5 & 6 & 7 & Ampicillin $^{+}$ \\
\hline $\begin{array}{l}\text { Acinetobacter } \\
\text { spp }\end{array}$ & $14 \pm 0.23$ & $7 \pm 0.0$ & $10 \pm 0.22$ & $14 \pm 0.24$ & $14 \pm 1.3$ & $9 \pm 0.0$ & $7 \pm 0.3$ & $14 \pm 0.14$ \\
\hline Escherichia coli & $12 \pm 0.12$ & $7 \pm 0.0$ & $10 \pm 0.22$ & $13 \pm 0.34$ & $15 \pm 2.0$ & $7 \pm 0.0$ & $7 \pm 1.0$ & $16 \pm 0.42$ \\
\hline $\begin{array}{l}\text { Klebsiella } \\
\text { Pneumonia }\end{array}$ & $14 \pm 1.24$ & $9 \pm 0.14$ & $10 \pm 0.14$ & $13 \pm 0.14$ & $12 \pm 1.3$ & $10 \pm 0.0$ & $7 \pm 1.0$ & $16 \pm 0.33$ \\
\hline $\begin{array}{l}\text { Pseudomonas } \\
\text { aeruginosa }\end{array}$ & $17 \pm 0.4$ & $7 \pm 0.00$ & $7 \pm 0.00$ & $13 \pm 0.22$ & $11 \pm 1.1$ & $7 \pm 0.0$ & $7 \pm 0.0$ & $18 \pm 0.24$ \\
\hline $\begin{array}{l}\text { Staphylococcus } \\
\text { aureus }\end{array}$ & $16 \pm 0.22$ & $7 \pm 0.0$ & $7 \pm 0.00$ & $11 \pm 0.33$ & $13 \pm 2.0$ & $7 \pm 0.0$ & $9 \pm 1.0$ & $26 \pm 0.22$ \\
\hline $\begin{array}{l}\text { Staphylococcus } \\
\text { epidermidis }\end{array}$ & $18 \pm 2.0$ & $7 \pm 0.0$ & $7 \pm 0.8$ & $11 \pm 0.22$ & $14 \pm 2.6$ & $10 \pm 1.3$ & $7 \pm 1.0$ & $34 \pm 4.20$ \\
\hline $\begin{array}{l}\text { Streptococcus } \\
\text { pneumonia }\end{array}$ & $16 \pm 0.8$ & $7 \pm 0.0$ & $10 \pm 0.2$ & $11 \pm 0.19$ & $15 \pm 3.1$ & $10 \pm 1.3$ & $7 \pm 1.4$ & $34 \pm 1.40$ \\
\hline
\end{tabular}


Table 2: MIC of compounds 1-7

\begin{tabular}{|c|c|c|c|c|c|c|c|c|}
\hline \multirow{2}{*}{ Test bacteria } & \multicolumn{8}{|c|}{ MIC ( $\mu \mathrm{g} / \mathrm{mL})$} \\
\hline & 1 & 2 & 3 & 4 & 5 & 6 & 7 & Ampicillin $^{+}$ \\
\hline $\begin{array}{l}\text { Acinetobacter } \\
\text { spp }\end{array}$ & $30^{*}$ & $\geq 30$ & $30^{*}$ & $30^{*}$ & $30^{*}$ & $\geq 30$ & $30^{*}$ & - \\
\hline Escherichia coli & $30^{*}$ & $\geq 30$ & $30^{*}$ & $30^{*}$ & $30 *$ & $\geq 30$ & $30^{*}$ & $2 \pm 0.2$ \\
\hline $\begin{array}{l}\text { Klebsiella } \\
\text { pneumonia }\end{array}$ & $30^{*}$ & $\geq 30$ & $30^{*}$ & $30^{*}$ & $30^{*}$ & $\geq 30$ & $30^{*}$ & $2 \pm 0.2$ \\
\hline $\begin{array}{l}\text { Pseudomonas } \\
\text { aeruginosa }\end{array}$ & $15^{*}$ & $\geq 30$ & $\geq 30$ & $30^{*}$ & $30^{*}$ & $\geq 30$ & $30^{*}$ & $3 \pm 0.2$ \\
\hline $\begin{array}{l}\text { Staphylococcus } \\
\text { aureus }\end{array}$ & $15^{*}$ & $\geq 30$ & $\geq 30$ & $30^{*}$ & $30^{*}$ & $\geq 30$ & $30^{*}$ & $5 \pm 0.4$ \\
\hline $\begin{array}{l}\text { Staphylococcus } \\
\text { epidermidis }\end{array}$ & $15^{\star}$ & $\geq 30$ & $\geq 30$ & $30^{*}$ & $30^{*}$ & $30^{*}$ & $30^{*}$ & $5 \pm 0.2$ \\
\hline $\begin{array}{l}\text { Streptococcus } \\
\text { pneumonia }\end{array}$ & $15^{*}$ & $\geq 30$ & $30^{*}$ & $30^{*}$ & $30^{*}$ & $30^{*}$ & $30^{*}$ & - \\
\hline
\end{tabular}

${ }^{+}$Ampicillin was used as control positive, *: significant difference at $p<0.05$

Table 3: Toxicity and antitumor activity of compounds 1-7

\begin{tabular}{|c|c|c|c|c|c|c|c|c|}
\hline \multirow{2}{*}{ Test } & \multicolumn{8}{|c|}{$\mathrm{LD}_{50}(\mu \mathrm{g} / \mathrm{mL})$} \\
\hline & 1 & 2 & 3 & 4 & 5 & 6 & 7 & Control $^{\mathrm{a}}$ \\
\hline $\begin{array}{l}\text { Toxicity against } \\
\text { A. salina }\end{array}$ & ND & ND & ND & $25^{*}$ & $\geq 250$ & $\geq 250$ & $\geq 250$ & 250 \\
\hline $\begin{array}{l}\text { Antitumor } \\
\text { against Erlich } \\
\text { carcinoma cells }\end{array}$ & ND & ND & ND & $50^{*}$ & 100 & $\geq 250$ & $\geq 250$ & 30 \\
\hline
\end{tabular}

${ }^{a}$ Quinidine sulfate used as positive control for toxicity assay and elspar (trade name for asparaginase) for antitumor assay, $N D=$ not detected; *significant difference at $p<0.05$

Compound $\mathbf{2}$ was isolated as colorless oil. A search of chemical database using science finder found that the spectroscopic data of $\mathbf{2}$ was in accordance with the chemical structure of nardol, isolated previously from the rhizome of Nardostachys jatamansi [24]. This is the first report of ${ }^{13} \mathrm{C}-\mathrm{NMR}$ from this compound.

Compound 3 was isolated as colorless oil. The resulted spectroscopic data (cf. exp.) gave conclusion that the compound similar to aristol(9)-ene, previously isolated from a soft coral Lemnalia humesi [25].

Compound 4 was isolated as colorless oil. 4 was found to be Cembrene $A$, a cembranoid previously isolated from the paracloacal glands of the Chinese Alligator (Alligator sinensis) [26].

Compound 5 was isolated as colorless oil. The spectroscopic data of 5 (Cf. exp.) was found to be stereochemically similar with the structure of alismoxide, a guaianediol with stereostructure $1 S^{*}, 4 S^{*}, 5 S^{*}, 10 R^{*}-4,10$-guaianediol, isolated previously from the rhizome of Alisma plantagoaquatu and soft coral Sinularia sp. [22,27].

Compound 6 was isolated as amorphous powder. The obtained spectroscopic data (cf. exp.) of 6 was found to be stereochemically similar with the structure of chalinasterol, a steroid isolated previously from a soft coral of Sinularia gibberosa and Lytophyton arboretum $[28,29]$.

Compound 7 was isolated as amorphous powder. A constructed structure based on the analysis of the spectral data (cf. exp.) was searched by science finder and found to be similar in structure with Nephalsterol C, a steroid isolated previously from a soft coral Lytophyton arboreum [29].

Biological activity assays confirmed that isoprenoids type compounds from Lobophytum sp. possessing antibacterial and antitumor activity. Compound 1 (alismol) has strong antibacterial activity against both gram positive (S. aureus, S. epidermis, and S. pneumonia) and negative bacteria ( $P$. aeruginosa). Nevertheless, the antitumor activity was not detected. Compound 4 (cembrene A) showed moderate activity against all tested bacteria. Moreover, it also possesed high toxicity $(25 \mu \mathrm{g} / \mathrm{mL})$ against $A$. salina and appreciable antitumor activity (50 $\mu \mathrm{g} / \mathrm{mL}$ ) against Erhlich carcinoma cells.

\section{CONCLUSION}

Four sesquiterpenes, one cembranoid type diterpene and two steroids have been isolated and identified from the Red Sea soft coral 
Lobophyton sp. Compound 1 (alismol) has significant antibacterial activity against grampositive ( $S$. aureus, $S$. epidermis, and $S$. pneumonia) and gram-negative bacteria ( $P$. aeruginosa). Compound 4 (cembrene $A$ ) also exhibited moderate antibacterial activity against all tested bacteria and appreciable antitumor properties against Erhlich carcinoma cells. Compounds 1 and 4 display potentials for treating pathogenic bacteria associated with many diseases and/or tumor and therefore, require further investigation

\section{DECLARATIONS}

\section{Acknowledgement}

The authors wish to thank Dr. Mohsen ElSherbiny, Researcher, Marine Biology Department, Faculty of Marine Sciences, King Abdulaziz University, for collection and identification of the soft coral sample.

\section{Conflict of Interest}

No conflict of interest associated with this work.

\section{Contribution of Authors}

The authors declare that this work was done by the authors named in this article and all liabilities pertaining to claims relating to the content of this article will be borne by them.

\section{REFERENCES}

1. Blunt JW, Copp BR, Keyzers R.A, Munro MHG, Prinsep MR. Marine natural products. Nat Prod Rep 2012; 29 . 144-222.

2. Al-Lihaibi SS, Alarif WM, Lateef AA, Ayyad SN, AbdelNaim AB, El-Senduny FE, Badria FA. Three new cembranoid-type diterpenes from Red Sea soft coral Sarcophyton glaucum: isolation and anti-proliferative activity against HepG2 cells. Eur J Med Cem 2014; 81 : 314-322.

3. Al-Footy KO, Alarif WM, Asiri F, Aly MM, Ayyad SEN. Rare pyrane-based cembranoids from the Red Sea soft coral Sarcophyton trocheliophorum as potential antimicrobial-antitumor agents. Med Chem Res 2015; 24(2): 505-512.

4. Lateff AA, Alarif WM, Ayyad SEN, Al-Lihaibi SS, Basaif $S A$. New cytotoxic isoprenoid derivatives from the Red Sea soft coral Sarcophyton glaucum. Nat Prod Res: Formerly Nat Prod Lett 2015; 29(1): 24-30.

5. Zubair M, Alarif W, Al-Footy K, PH M, Aly M, Basaif S, AlLihaibi S, Ayyad SE, New antimicrobial biscembrane hydrocarbon and cembranoid diterpenes from the soft coral Sarcophyton trocheliophorum. Turk J Chem 2016; 40(3): 385-392.

6. Wang SK, Duh CY, Wu YC, Wang Y, Cheng MC, Soong $K$, Fang LS. Studies on Formosan Soft Corals, II. Cytotoxic Cembranolides from the Soft Coral Lobophytum michaelae. J Nat Prod 1992; 55: 1430 1435.

7. Coval SJ, Patton RW, Petrin JM, James L, Rothofsky ML, Lin SL, Patel M, Reed JK, McPhil AT, Bishop WRA. A cembranolide diterpene farnesyl protein transferase inhibitor from the marine soft coral Lobophytum cristagalli. Bioorg Med Chem Lett 1996; 6: 909-912.

8. Higuchi R, Miyamoto $T$, Yamada K, Komori T. Cytotoxic and ichthyotoxic compounds from marine opisthobranchia and soft coral. Toxicon 1998; 36: 1703-1705.

9. Matth'e GF, König GM, Wright AD. Three New Diterpenes from the Marine Soft Coral Lobophytum crassum. J Nat Prod 1998; 61: 237-240.

10. Duh CY, Wang SK, Huang BT, Dai CF. CytotoxiC Cembrenolide Diterpenes from the Formosan Soft Coral Lobophytum crassum, J Nat Prod 2000; 63:884-885.

11. Chao CH, Hang HC, Wu YC, Lu CK, Dai CF, Sheu JH. Glycolipids from the Formosan soft coral Lobophytum crassum. Chem Pharm Bull 2007; 55: 1720-1723.

12. Zhang W, Krohn K, Ding J, Miao ZH, Zhou XH, Chen SH, Pescitelli G, Salvadori P, Kurtan T, Guo YW. Structural and stereochemical studies of $\alpha$-methylene- $\gamma$-lactonebearing cembrane diterpenoids from a South China Sea soft coral, J Nat Prod 2008; 71: 961-966.

13. Lin ST, Wang SK, Cheng SY, Duh CY. Lobocrasol, a new diterpenoid from the soft coral Lobophytum crassum. Org Lett 2009; 14: 3012-3014.

14. Cheng SY, Wen ZH, Chiou SF, Wang SK, Hsu CH, Dai CF, Chiang MY, Duh CY. Durumolides A-E, Antiinflammatory and antibacterial cembranolides from the soft coral Lobophytum durum. Tetrahedron 2008; 64 : 9698-9704.

15. Cheng SY, Wen ZH, Wang SK, Chiou SF, Hsu CH, Dai $C F$, Duh CY. Anti-inflammatory cembranolides from the soft coral Lobophytum durum. Bioorg Med Chem 2009; 17: 3763-3769.

16. Cheng SY, Wen ZH, Chiou SF, Wang SK, Hsu CH, Dai CF, Chiang MY, Duh CY. Unprecedented hemiketal cembranolides with anti-inflammatory activity from the soft coral Lobophytum durum. J Nat Prod 2009; 72 : 152-155.

17. Rashid MA, Gustafson KR, Boyd MR. HIV-inhibitory cembrane derivatives from a Philippines collection of the soft coral Lobophytum species. J Nat Prod 2000; 63: 531-533.

18. Holder IA, Boyce ST. Agar well diffusion assay testing of bacterial susceptibility to various antimicrobials in concentrations non-toxic for human cells in culture. Burns 1994; 20: 426-429.

19. Chand S, Lusunzi I, Veal DAL, Williams R, Karuso $P$. Rapid screening of the antimicrobial activity of extracts and natural products. J Antibiotics 1994; 47: 1295-1304. 
20. Aly MM, Gumgumjee N. Antimicrobial efficacy of Rheum palmatum, Curcuma longa and Alpinia officinarum extracts against some pathogenic microorganisms. Afr $\mathrm{J}$ Biotechnol 2011; 10: 12058-12063.

21. Meyer BN, Ferrigni NR, Putnam JE, Jacobsen LB, Nichols DE, Mclaughlan JL. Brine shrimp: a convenient general bioassay for active plant constituents. Plant Med 1982; 45: 31-34.

22. Oshima $Y$, Iwakawa T, Hikino H. Alismol and alismoxide, sesquiterpenoids of Alisma rhizomes. Pytochem 1983, 22: 183-185.

23. Penga GP, Tiana G, Huanga XF, Loub FC. Guaiane-type sesquiterpenoids from Alisma orientalis. Phytochem 2003; 63: 877-881.

24. Sastry SD, Maheshwari ML, Bhattacharyya SC. Terpenoids LXXXVII. The structure of nardo/*. Tetrahedron Letter 1966; 10: 1035-10121
25. Bowden BF, Coll JC, Mitchell SJ. Studies of Australian Soft Corals. XVI*Two New Sesquiterpenes from Lemnalia humesi. Aust J Chem 1980; 33: 681-684.

26. Mattern DL, Scott WD, McDaniel CA, Weldon PJ, Graves $D E$. Cembrene $A$ and a Congeneric Ketone Isolated from the Paracloacal Glands of the Chinese Alligator (Alligator sinensis). J Nat Prod 1997; 60: 828-831.

27. Zhang GW, Ma XQ, Su JY, Zhang K, Kurihara $H$, Yao $X S$, Zeng $L M$, Two new bioactive sesquiterpenes from the soft coral Sinularia sp. Nat Prod Res 2006; 20(7): 659-664.

28. Grote D, Dahse HM, Seifert K, Furanocembranoids from the soft corals Sinularia asterolobata and Litophyton arboretum. Chem Biodivers 2008; 5: 2449-2456.

29. Ellithey MS, Lall N, Hussein AA, Meyer D. Cytotoxic, Cytostatic and HIV-1 PR Inhibitory Activities of the Soft Coral Litophyton arboretum. Mar Drugs 2013; 11: 49174936. 